\title{
MOTIVACIÓN PARA EL TRABAJO INTELECTUAL, ESTILOS DE APRENDIZAJE Y ESTRATEGIAS METACOGNITIVAS EN ALUMNOS DE EDUCACIÓN MEDIA
}

\section{MOTIVATION FOR INTELLECTUAL WORK, LEARNING STYLES AND METACOGNITIVE STRATEGIES IN MIDDLE SCHOOL STUDENTS}

\author{
Jorge Omar Trisca \\ Universidad de Montemorelos, México \\ trisca@um.edu.mx \\ Anneth Medina Rocha \\ Universidad de Montemorelos, México \\ annethmr@gmail.com \\ Jaime Rodríguez Gómez \\ IUNIS, México \\ jar@um.edu.mx \\ Mónica Cely Salazar \\ Fundación Universitaria Konrad Lorenz, Colombia \\ ps.monicacely@gmail.com
}

\begin{abstract}
RESUMEN
El aprendizaje autorregulado, los estilos de aprendizaje y la motivación para el trabajo intelectual tienen gran importancia en la actualidad por su influencia sobre los procesos de aprendizaje. Este estudio, descriptivo y correlacional, procura determinar el grado de relación que existe entre estas variables y su nivel de significación, pues esta información podría ser de gran ayuda en el momento de planear las estrategias de aprendizaje dentro de las aulas. Se administró el Cuestionario Honey-Alonso de Estilos de Aprendizaje (CHAEA) y el Cuestionario de Estrategias de Aprendizaje y Motivación (CEAM) para el trabajo intelectual a una muestra de 110 alumnos de una escuela preparatoria de Mérida, Yucatán, México. Se encontró que la supervisión y la autoevaluación, como estrategias metacognitivas, fueron las mejores valoradas. Por otro lado, la organización de la información resaltando las ideas principales obtuvo una valoración muy pobre. El modelo planteado para analizar la relación entre las estrategias metacognitivas, la motivación y los estilos de aprendizaje alcanzó niveles de ajuste satisfactorios. Se observa una aparente ausencia de relación entre
\end{abstract}


la motivación y los estilos de aprendizaje en coincidencia con resultados de otros estudios consultados. Por otro lado, en la literatura se ha encontrado que estudiantes con ciertos estilos de aprendizaje tienden a tener una preferencia por determinadas estrategias metacognitivas. No obstante, en la presente investigación no se observaron tales correlaciones, lo cual puede atribuirse a que en esta investigación el desarrollo de los diferentes estilos de aprendizaje es muy equilibrado, además de que únicamente la mitad de los encuestados tiende a tener un estilo preferente. En conclusión, los estilos de aprendizaje conforman un constructo que es independiente de la motivación y las estrategias metacognitivas.

Palabras clave: motivación, estilos de aprendizaje, metacognición, autorregulación

\section{ABSTRACT}

Self-regulated learning, learning styles, and motivation for intellectual work are of great importance today because of their influence in learning processes. This descriptive and correlational study seeks to discern the relational degree that exists between these variables and their level of significance, as this information could be of great help when planning learning strategies within the classroom. The Honey-Alonso Learning Styles Questionnaire and the Learning and Motivation Strategies for Intellectual Work Questionnaire) were administered to a sample of 110 students from a high school in Mérida, Yucatan, Mexico. It was found that supervision and self-evaluation, as metacognitive strategies, were the most valued. On the other hand, the organization of information highlighting the main ideas obtained a very poor assessment. The model proposed to analyze the relationship between metacognitive strategies, motivation, and learning styles reached satisfactory levels of adjustment. There is an apparent lack of relationship between motivation and learning styles, which is in coincidence with results from other studies. On the other hand, in the literature, it has been found that students with particular learning styles tend to have a preference for specific metacognitive strategies. However, in the current research, no such correlations were observed, which can be attributed to the fact that in the said study, the development of the different learning styles was very balanced, in addition to the fact that only half of the respondents tended to have a preferred style. In conclusion, learning styles make up a construct that is independent of motivation and metacognitive strategies.

Keywords: motivation, learning styles, metacognition, self-regulation

\section{Introducción}

Existen múltiples variables que inciden en los procesos académicos de los alumnos. Entre las más importantes se encuentran el aprendizaje autorregulado, los estilos de aprendizaje y la motivación para el trabajo intelectual. En esta investigación se intentó encontrar relaciones significativas entre esas variables en estudiantes de nivel medio en un rango de edad de 12 a 18 años. En la literatura se encontró una profusa 
producción de investigación para cada una de las variables y algo menos acerca de las relaciones entre ellas. Por tal razón, y para un mejor entendimiento de la temática, se procedió a un análisis de las variables estudiadas y de las relaciones entre ellas. También se describieron algunas variables asociadas con las variables del estudio, tales como las estrategias metacognitivas y la metacognición.

\section{Motivación}

La motivación es entendida, desde la perspectiva de Morales Rodríguez (2011), como un proceso que facilita la activación, dirección y persistencia de la conducta, mientras que Maggiolini (2013) la considera como el deseo o necesidad. De acuerdo con la propuesta de Morales Rodríguez (2011), la motivación dirige la conducta hacia una meta. En este proceso se considera la expectativa como un elemento orientador de la conducta, la cual permite connotar el valor asignado a la meta por parte de la persona. Se puede afirmar, entonces, que la motivación no sólo es causa, sino también efecto, logra la activación de la conducta y la dirige hacia la consecución de una meta, permitiendo que el sujeto tenga claro el objetivo y emplee los recursos cognitivos, emocionales y comportamentales para llegar a dicho fin.

La motivación involucra una serie de constructos, como lo son las intenciones, las metas, las percepciones y las creencias (Pool-Cibrián y Martínez-Guerrero, 2013). Estos constructos se reconocen como representaciones mentales que permiten comprender la interacción continua que se da entre ámbito cognitivo y afectivo (Morales Rodríguez, 2011). La motivación logra "elicitar cogniciones y emociones con respecto a las actividades de aprendizaje" (Suárez Riveiro y Fernández Suárez, 2013, p. 233).

Existen diversas teorías motivacionales. Una de ellas es la relacionada con la intención y la fuente motivacional. Desde el modelo sociocognitivo, que vincula los constructos de poder, afiliación y logro, el poder implica la necesidad de controlar el comportamiento de los demás; la afiliación, se relaciona con la necesidad de ser miembros de algo; y el logro con la necesidad de alcanzar una meta (Maggiolini, 2013). La fuente motivacional, por su parte, invita a reconocer dónde se origina el deseo por alcanzar determinado logro. Cuando el interés se da por la actividad en sí misma, esta es vista como un fin y a este proceso se le denomina motivación intrínseca. La motivación extrínseca se relaciona con factores externos. En los contextos académicos implica la necesidad de aprobar o el interés por obtener buenas calificaciones (Rinaudo, Chiecher y Donolo, citados en Herczeg y Lapegna, 2010).

La motivación en el ámbito académico es concebida por López Aguado y Silva Falchetti (2009) como la disposición que lleva al estudiante a implicarse en el proceso de aprendizaje, esta puede cambiar en función de aspectos sociales o ambientales. Podría relacionarse este factor con aspectos pedagógicos como la inclusión de estrategias de enseñanza basadas en las TICs por parte de los docentes, las cuales pueden incidir en la activación que presentan los estudiantes durante el desarrollo de las sesiones de clase. Como afirma Dewey (1984, citado en Maggiolini, 2013), siempre que una actividad estuviera centrada en un eje de interés, la participación de los alumnos estaría garantizada. 
Contar con creencias de control del aprendizaje favorece también la motivación y el rendimiento escolar. La valoración positiva de las tareas por parte del estudiante así como las creencias de autoeficiencia favorecen la motivación, dado que generan cambio en el locus de control interno (Herczeg y Lapegna, 2010). Rotter (1966, citado en Rinaudo, Chiecher y Donolo, 2003) llama "locus de control interno" al proceso que permite percibir que el control sobre una tarea está en la persona y "locus de control externo" a la percepción de que el control está en manos de otra persona, o es responsabilidad de otros.

La motivación incide principalmente en el esfuerzo y la persistencia del estudiante, permitiéndole actuar sobre la ejecución de las tareas y por ende incidir sobre su rendimiento (Suárez Riveiro y Fernández Suárez, 2013. En cuanto a la forma en la cual se realizan las tareas académicas, se identifica que "los estudiantes pueden de forma activa, adaptar o cambiar sus estrategias en función de factores tanto personales como contextuales y pueden aprender nuevas estrategias [de autorregulación motivacional]" (Suárez Riveiro, Fernández Suárez, Rubio Sánchez y Zamora Menéndez, 2016, p. 422). Estos factores pueden relacionarse con los aspectos evaluados en la prueba como las concentraciones-distracciones y la ansiedad-miedo al fracaso. Indudablemente, la forma en la cual el estudiante activa su conducta para el desarrollo de una actividad académica trae consigo la atención o no de aspectos ambientales que puedan distraerlo, así como la evitación al fracaso.

Se resalta el planteamiento de metas, ya que estas pueden ser de dos grandes tipos, como lo proponen Rodríguez, Piñeiro, Regueiro, Gayo y Valle (2014), las que se relacionan con el dominio y las de rendimiento. En las primeras, los estudiantes tienden a utilizar procesos cognoscitivos complejos, mientras que en las segundas los estudiantes se orientan a demostrar sus capacidades y competencias. Las metas vinculadas al rendimiento se relacionan con diversos motivos, tales como la búsqueda social, de recompensas externas o de evitación de castigos. Pool-Cibrian y Martínez-Guerrero (2013) han observado que las metas se convierten en guías en el proceso de autorregulación, permiten seleccionar estrategias de organización, planeación, manejo del tiempo, búsqueda de ayuda y comprensión de temas.

Ahora bien, estudios como el realizado por Morales Rodríguez (2011) dan cuenta de la incidencia de la motivación en aspectos cognitivos como las expectativas, las atribuciones, los motivos y los intereses, y puede determinar el éxito o no de las estrategias implementadas. En consecuencia, se puede afirmar que la motivación académica se relaciona con todo el proceso académico del estudiante, desde el inicio, su perseverancia y las acciones que permiten el aprendizaje. A partir del control y de la conciencia motivacional que se consigue mediante la autoobservación y el autoconocimiento, el aprendiz autorregulado es capaz de detectar los desajustes y controlar sus emociones mediante una serie de estrategias con el fin de regular su motivación. Tales estrategias son conocidas como estrategias de regulación de la motivación (Pintrich y Wolters, citados en Suárez Riveiro y Fernández Suárez, 2013). Uno de los aspectos más importantes de estas estrategias es que pueden ser adoptadas conscientemente o bien ponerse en marcha de forma automática, 
pero en todo caso, pueden ser enseñadas y aprendidas, y consecuentemente, modificadas. Aunque en este modelo no se hace referencia explícita a cuestiones volitivas, que tanto se han estudiado en la motivación, sobre todo aspectos como la persistencia y el esfuerzo (Rodríguez, Piñeiro, Regueiro, Gayo y Valle, 2014), Pintrich las incluye en las estrategias de regulación de la motivación (Valle et al., 2010).

\section{Aprendizaje autorregulado}

Las estrategias metacognitivas forman parte de un constructo mayor denominado aprendizaje autorregulado o metacognición. En efecto, de acuerdo con la literatura consultada (Lanz, 2006; Torrano Montalvo y González Torres, 2004), el estudio del aprendizaje autorregulado ha tenido un gran auge en los últimos años. Sin embargo, históricamente la delimitación de su campo de estudio ha tendido a ser un tanto difuso. De hecho, conceptos como autorregulación y metacognición tienen límites imprecisos e incluso se los llega a tomar como sinónimos (Lanz, 2006). Es probable que la falta de fronteras claras se deba a que todos los elementos forman parte de un mismo proceso, esto es, tratar de descifrar cómo el sujeto conoce, regula y produce su propio aprendizaje. Otra posible razón sea que, debido a la gran cantidad de investigaciones al respecto, se ha saturado el concepto, por lo que es complicado delimitarlo (Rosário et al., 2014). En este contexto, Paris y Winograd (citados en Herczeg y Lapegna, 2010) plantean que el aprendizaje autorregulado se caracteriza por el conocimiento acerca del propio conocimiento (metacognición), el cual favorece la selección de estrategias para promover la efectividad en el aprendizaje, el uso de las estrategias y el reconocimiento de los momentos y circunstancias en las cuales es más favorable la aplicación de las mismas (estrategias metacognitivas) y la motivación.

En tanto, Valle et al. (2010) sugieren que la noción de aprendizaje autorregulado surge de conceptualizar al aprendizaje como la construcción de conocimiento, donde el estudiante debe ser consciente de los conocimientos que posee y de las estrategias que requiere para aprender, y planear las estrategias que facilitarán la construcción del conocimiento. Zimmerman y Schunk (1989, citados en Valle et al., 2010) definen el aprendizaje autorregulado como un proceso en el que los pensamientos, sentimientos y acciones son autogeneradas por los estudiantes y afecta a los aspectos cognitivo, afectivo-motivacional y comportamental. En líneas generales, puede decirse que el aprendizaje autorregulado incluye las estrategias cognitivas, la metacognición y la motivación, entre otros elementos (Lamas Rojas, 2008).

Flavell, Nisbet y Schucksmith (citados en Herczeg y Lapegna, 2010) introducen el término "metacognición" para referirse "al conocimiento que tiene un individuo sobre cualquier aspecto de una tarea cognitiva y a la regulación que es capaz de hacer sobre esos aspectos" (p. 10). De hecho, tampoco hay un acuerdo entre los investigadores sobre algunos aspectos más borrosos de la metacognición, pero parece haber aceptación mayoritaria que una definición de metacognición debería incluir al menos estas nociones: conocimiento, por parte del sujeto, de su propio conocimiento, procesos y estados cognoscitivos y afectivos, y capacidad de supervisarlos y regularlos deliberadamente (Hacker, 1995). 
De manera que, tanto para el aprendizaje autorregulado como para la noción de metacognición, aparecen prácticamente los mismos factores intervinientes. Con todo, el concepto de aprendizaje autorregulado incorpora el factor motivacional (Valle et al., 2010), por lo que enriquece aun más el constructo.

Partiendo entonces de los componentes que influyen en el aprendizaje autorregulado, las estrategias metacognitivas aparecen como un subgrupo de los procesos autorregulatorios. Las estrategias metacognitivas están ampliamente relacionadas con el aprendizaje autorregulado e incluyen las estrategias referidas a la planificación, supervisión y regulación de la cognición (Suárez Riveiro y Fernández Suárez, 2013).

Las estrategias metacognitivas pueden agruparse en tres dimensiones: autoconocimiento, autorregulación y evaluación (Arias Gallegos, Zegarra Valdivia y Justo Velarde, 2014). Estas estrategias han sido organizadas de manera diferente dependiendo de los investigadores y son fundamentales para el aprendizaje académico, de acuerdo con las investigaciones llevadas a cabo al respecto (Suárez Riveiro y Fernández Suárez, 2013). Desde luego, no solo influyen las estrategias metacognitivas en el aprendizaje autorregulado; también aspectos como la noción de autoeficacia, la adopción de metas, el interés y la valorización de la tarea contribuyen al logro académico (Valle et al., 2010), pero parece fundamental que los déficit en los procesos de autorregulación se relacionan principalmente con la ineficacia para planear y controlar las tareas y actividades académicas (Pool-Cibrian y Martínez-Guerrero, 2013), aspectos que están directamente relacionados con las estrategias metacognitivas.
Maris Vázquez, Noriega Biaggio y Maris García (2013) sostienen que, en general, hay acuerdo en que los estudiantes autorregulados dirigen su aprendizaje mediante el uso de estrategias cognitivas, metacognitivas, motivacionales y de apoyo, de modo que pueden controlar y regular intencionalmente su proceso de aprendizaje. Esto implica un conocimiento acabado de sus habilidades personales, los conocimientos que tienen, y lo que deben hacer para aprender, además de haber aprendido a monitorear sus avances cuando estudian y a predecir las dificultades de las diferentes tareas académicas. A lo anterior, se suma la motivación personal que le permite al estudiante regular sus esfuerzos y controlar los factores internos y externos que pueden afectar su rendimiento.

\section{Estilos de aprendizaje}

Tripodoro y De Simone (2015) establecen que las premisas de la educación actual fueron fundadas bajo un supuesto que valoraba el conocimiento, la obediencia, la adaptación al modelo y la "pasividad" del alumno en la tarea de adquirir el conocimiento transmitido. Las nuevas estrategias de enseñanza han tenido que romper algunos paradigmas, entre ellos, que la capacidad intelectual no es, por sí sola, un factor definitorio para el rendimiento académico y, por otro lado, en quién debe estar centrada la educación (Maris Vázquez et al., 2013).

En las instituciones escolares suelen encontrarse al menos dos tipos de enseñanza, una centrada en el profesor y otra en el estudiante. El enfoque predominante depende totalmente del profesor, quien podría tener una formación en docencia o no, y, aunque al parecer los estudios respaldan que conviene una educación centrada en el estudiante, la 
mayoría de las instituciones no la desarrollan (Laudadío y Da Dalt, 2014). Sin embargo, el enfoque educativo se ha transformado, convirtiendo al profesor en un agente facilitador de conocimiento, que toma en cuenta el papel del estudiante para ese proceso de aprendizaje en el que está constantemente desarrollando habilidades (Aguilera Pupo y Ortiz Torres, 2008).

Conscientes del papel activo del estudiante, se ha vuelto primordial el estudio de los procesos de su autogestión, donde cobran importancia los estilos de aprendizaje, que caracterizan a cada alumno en su forma de aprender. "Son definidos como operaciones cognitivas básicas, que se integran a patrones de personalidad y que permiten la interacción del sujeto con el entorno, es una forma de interacción sociocognitiva. Son procedimientos generales de aprendizaje" (Isaza Valencia, 2014, p. 27). Una de las teorías más utilizadas es la de Kolb, propuesta que permite entender aspectos como las necesidades y motivaciones del individuo (Arias Gallegos et al., 2014). Respecto a la motivación, el docente puede impactar en la relación de motivos intrínsecos y extrínsecos, donde se puede generar el deseo de aprender, para que el alumno haga suyos los motivos que percibía fuera de él, estimulando así el proceso de aprendizaje (Aguilera Pupo y Ortiz Torres, 2008). No obstante, esto no significa que exista una relación significativa entre los estilos de aprendizaje y la motivación.

La teoría de Kolb propone un método que describe la forma en que los estudiantes logran resolver problemas y aplicar sus aprendizajes a la experiencia personal. Su teoría es holística y explora perfiles que abordan la experiencia concreta, la observación reflexiva, la conceptualización abstracta y la experimentación activa. Las dos primeras están orientadas a la manera en que se percibe la información y las otras dos a la manera de procesar la información (Arias Gallegos et al., 2014). Para valorar la caracterización de los perfiles de estilos de aprendizaje se deben tomar en consideración al menos tres dimensiones: afectiva, cognitiva y metacognitiva (Aguilera Pupo y Ortiz Torres, 2008). López Aguado y Silva Falchetti (2009) creen que la propuesta de Kolb obedece a una comprensión del aprendizaje desde la perspectiva experiencial. Kolb propone que, cuantos más estilos de aprendizaje domine el alumno, este tendrá una mayor retención de la información. Los estilos de aprendizaje surgen cuando se combinan las maneras de percibir la información y de procesarla, dando como resultado los cuatro principales estilos de aprendizaje (Arias Gallegos et al., 2014). Desde esta concepción, los cuatro estilos de aprendizaje derivados de la teoría de Kolb y desarrollados por Honey y Mumford son los siguientes: (a) estilo activo, orientado al reconocimiento de nuevas ideas, emprendimiento de acciones innovadoras; (b) estilo reflexivo, orientado a realizar análisis en profundidad; (c) estilo teórico, orientado a comprender las experiencias desde marcos teóricos explicativos y (d) estilo pragmático, orientado a la puesta en práctica de las ideas abstractas (López Aguado y Silva Falchetti, 2009).

En estudios realizados sobre la relación que tienen los estilos de aprendizaje con el aprendizaje autorregulado no se encontró una correlación significativa, pero se observó una correlación entre la carrera y la preferencia de los estilos de aprendizaje de los alumnos (Arias Gallegos et al., 2014; Sanfabián 
Maroto, Belver Domínguez y Álvarez Álvarez, 2014).

La influencia de los estilos de aprendizaje sobre el rendimiento académico ha sido discutida, pues algunos autores han hallado una correlación positiva entre estas variables (Blumen, Rivero y Guerrero, 2011; Kohler Herrera, 2013) mientras que otros no encuentran correlación (Sanfabián Maroto et al., 2014). También se ha percibido una correlación entre el estilo de aprendizaje teórico y el aprovechamiento, pues este estilo tiende a exhibir mejores resultados en el rendimiento académico (Blumen et al., 2011). Los estilos de aprendizaje pragmáticos y teórico se asocian con modelos tradicionales de enseñanza y son los que en su mayoría dominan los estudiantes de educación superior (Isaza Valencia, 2014).

Asimismo, en un programa de formación híbrida que incluía estrategias de enseñanza que consideraban los diferentes estilos de aprendizaje, se encontró una mejora significativa en el desempeño de los alumnos de todos los estilos de aprendizaje en las pruebas de conocimiento (Wichadee, 2013).

Aguilera Pupo y Ortiz Torres (2008) estudiaron la preferencia que tienen los alumnos en cuanto a los estilos de aprendizaje y sostienen que es importante considerar los objetivos normativos de la carrera para la caracterización de perfiles y el desarrollo de habilidades del estudiante. También consideran importante establecer vínculos interdisciplinarios a partir del contenido de las materias que permita ofrecer fundamentos psicológicos, pedagógicos y didácticos para que comprendan su estilo de aprendizaje y se responsabilicen de su proceso de aprendizaje.

Según Contreras Gastélum y Lozano
Rodríguez (2012), el avance del alumno en su carrera influye en el grado de autorregulación, mientras que la carrera influye significativamente en la preferencia de los alumnos por el estilo de aprendizaje de experimentación activa. Aunque por otra parte se ha encontrado un mismo patrón de preferencia de estilo de aprendizaje independientemente del avance de carrera.

\section{Relación entre las variables de estudio}

Como el estilo de aprendizaje es un constructo que permite comprender cómo las personas perciben el mundo, procesan la información, privilegian su proceso de aprendizaje y la instrucción, se lo ha relacionado con la motivación, en tanto esta permite orientar sus elecciones. Cázares Castillo (2009) ha identificado una estrecha relación entre la motivación intrínseca y los estilos de aprendizaje, pues estos últimos involucran procesos cognitivos, afectivos y fisiológicos. Los procesos afectivos de los estilos de aprendizaje implican activar, mantener y dirigir el comportamiento de una persona hacia determinada fuente de información y estrategia de procesamiento de información que resulte más satisfactoria y sea percibida por el sujeto como más efectiva.

Respecto a la relación que existe en las variables motivación, el aprendizaje autorregulado y estilos de aprendizaje, las primeras dos son las que han sido frecuentemente asociadas en la literatura consultada. De hecho, parece que la autorregulación no actúa independientemente, sino que se ve influenciada de manera directa por mecanismos relacionados con la motivación (Herczeg y Lapegna, 2010). 
El aprendizaje autorregulado implica una motivación que impulsa decisiones basadas en lo referente al objetivo de una actividad, la percepción de la dificultad y el valor de la tarea, las autopercepciones del alumno en cuanto a su capacidad para lograr la tarea, el beneficio potencial del éxito o la responsabilidad del fracaso (Paris y Winograd, 2001).

La motivación se relaciona con la autorregulación en tanto que incorpora la valoración del aprendizaje, la fijación de metas y la creencia positiva acerca de las capacidades personales. La concepción mediante la cual se integra el aprendizaje con componentes motivacionales, cognitivos y metacognitivos ha cobrado hoy en día un gran valor. Se identifica, por ejemplo, que los estudiantes que presentan problemas en el aprendizaje no cuentan con motivación intrínseca y por ende dependen por completo de aspectos externos para activar conductas que faciliten el aprendizaje (Alarcón Díaz, 2013).

Núñez Alonso, Lucas, Navarro Izquierdo y Grijalbo Lobera (2006) han encontrado que los alumnos autorregulados se caracterizan por su motivación e implicación personal en el aprendizaje y son capaces de persistir y esforzarse en las tareas para conseguir las metas que se han propuesto. Se considera que los alumnos autorregulados muestran un conjunto de actitudes y creencias adaptativas que los llevan a implicarse y persistir en las tareas académicas. Así, estos estudiantes se consideran muy eficaces y se centran en incrementar su nivel de aprendizaje valorando el material que tienen que asimilar como interesante, valioso y útil de aprender (Pan et al., 2013).

En este contexto, "las estrategias cognitivas son comportamientos que ayudan a adquirir información e integrarla al conocimiento ya existente, así como a recuperar la información disponible" (Herczeg y Lapegna, 2010, p. 13). Suárez Riveiro y Fernández Suárez (2013) utilizan un modelo que permite evidenciar cómo las estrategias motivacionales relacionadas con el componente de afectividad inciden sobre las estrategias cognitivas. Lamas Rojas (2008) plantea que puede considerarse autorregulado un estudiante, en la medida en que sea activo en su propio proceso de aprendizaje, lo cual se manifiesta en la construcción de metas, elaboración de planes para alcanzarlas, ejecutar dichas acciones y realizar su evaluación. Así "la motivación incide en lo que piensa una persona y por ende incide en su aprendizaje" (Alarcón Díaz, 2013, p. 3). La motivación, por su parte, se ha encontrado relacionada también con las estrategias de aprendizaje y la metacomprensión.

$\mathrm{Al}$ analizar la relación existente entre los estilos de aprendizaje, la metacognición y sus dimensiones, se encontró que existe relación entre el estilo de aprendizaje convergente y la metacognición (Arias Gallegos et al., 2014). También se ha encontrado relación con el estilo de aprendizaje teórico por el fuerte impacto que puede tener sobre la naturaleza de rasgos cognitivos, fisiológicos y afectivos propios del estudiante.

Se ha encontrado una relación estadísticamente significativa entre la motivación intrínseca y el estilo teórico, específicamente en las tareas de búsqueda y selección de la información (Honey y Mumford, 1986) Desde el estilo teórico el estudiante se interesará por ahondar en la información y promover habilidades cognitivas de organización, elaboración y transformación. 
Al parecer, los estudiantes con estilo de aprendizaje teórico y reflexivo hacen un mayor uso de estrategias metacognitivas que quienes tienen los estilos pragmático y activo (Gravini Donado y Iriarte Diazgranados, 2008). El estilo de aprendizaje teórico correlaciona positiva y significativamente con la estrategia metacognitiva de búsqueda de información. También se encontró que el estilo activo correlaciona positivamente con orientación intrínseca, conocimiento condicional, estrategias de manejo de información y conocimiento declarativo. Por su parte, el estilo pragmático correlaciona positivamente con el valor de la tarea. Finalmente, el estilo reflexivo correlaciona negativamente con conocimiento declarativo (Cázares Castillo, 2009).

Respecto al género, existen diferencias en cuanto a los estilos de aprendizaje entre mujeres y hombres. Las mujeres hacen un mayor uso de estrategias metacognitivas y procesamiento profundo de información, organizan mejor su ambiente de estudio y manejan mejor el tiempo (Vázquez, Noriega Biaggio y García, 2013).

Por otra parte, se ha encontrado evidencia de que en grupos donde se analizan los cuatro principales estilos de aprendizaje se pueden exhibir alta y baja frecuencia de uso de estrategias metacognitivas y que las estrategias, no los estilos de aprendizaje, pueden afectar el rendimiento en ciertos ejercicios educativos. Se investigó también, con estudiantes universitarios, si existía una relación entre los estilos de aprendizaje, la alta frecuencia de uso de estrategias metacognitivas en la efectividad al realizar ciertas tareas, pero solo se encontró una relación significativa en el manejo del tiempo y no en las demás variables (Pujol, 2008).

\section{Metodología}

El diseño de este trabajo ha sido organizado como una investigación empírica, cuantitativa y correlacional. Se recurrió a una muestra de 110 estudiantes de educación media desde el primer año de educación secundaria hasta el tercer año de preparatoria, que incluyó estudiantes de 12 a 18 años, de una escuela de Mérida, Yucatán, México, quienes contestaron anónima y voluntariamente el Cuestionario Honey-Alonso de Estilos de Aprendizaje (CHAEA) y el Cuestionario de Estrategias de Aprendizaje y Motivación (CEAM) para el trabajo intelectual en línea. Los resultados se capturaron automáticamente en una base de datos en línea para su posterior análisis.

Las estrategias de aprendizaje se valoraron mediante el CEAM, que utiliza una escala Likert de siete puntos. El instrumento se divide en las subescalas de motivación y estrategias metacognitivas. La motivación incluye los factores de ambiente social, concentración, motivos para estudiar y ansiedad o miedo al fracaso. Las estrategias metacognitivas evaluadas incluyen la planificación para el aprendizaje, supervisión y evaluación, selección de la información, organización de la información y elaboración de relaciones comprensivas.

Para la medición de los estilos de aprendizaje, se utilizó la escala dicotómica del CHAEA. El instrumento valora los estilos activo, reflexivo, teórico y pragmático. En ambas escalas, un mayor puntaje implica, respectivamente, un mayor uso de la estrategia y una mayor cantidad de características asociadas a uno de los estilos. 


\section{MOTIVACIÓN PARA EL TRABAJO INTELECTUAL}

\section{Resultados}

Un análisis descriptivo de las estrategias de aprendizaje y motivación permite observar que la estrategia supervisión y autoevaluación fue la mejor valorada (ver Tabla 1). Los alumnos tienden a revisar lo que han fallado después de un examen $(M=5.5, D E=1.67)$, hacerse preguntas sobre el tema que están estudiando $(M=5.4, D E=1.66)$ y ver si sus trabajos se ajustan a lo solicitado por sus maestros $(M=5.4, D E=1.63)$. Por otro lado, la organización de la información resaltando las ideas principales es muy pobre: no destacan las ideas principales en sus apuntes $(M=2.6, D E=1.50)$, no encuentran ayuda en organizar las ideas antes de escribir $(M=2.7, D E=1.73)$ y no les parece imprescindible identificar las ideas principales antes de memorizar contenidos sobre un tema $(M=3.0, D E$ $=1.33)$.

En la motivación, resaltan los motivos para estudiar (ver Tabla 1), de tal forma que ven en el estudio una preparación para un futuro interesante $(M=$ $6.4, D E=1.22$ ), se sienten estimulados cuando sus profesores les felicitan $(M=6.0, D E=1.36)$ y se esfuerzan por conseguir buenas notas para poder escoger la profesión que les interesa $(M=5.7, D E=1.43)$. Sin embargo, hay cierto temor de no terminar sus estudios para las profesiones que les gustan $(M=4.8, D E=2.15)$, a pesar de que piensan que si se proponen algo son capaces de conseguirlo $(M=6.0$, $D E=1.22)$, ya que han superado los cursos anteriores con facilidad ( $M=$ 5.2, $D E=1.72)$.

\section{Tabla 1}

Descriptivos de las variables observadas

\begin{tabular}{lcccccc}
\hline \multicolumn{1}{c}{ Variable } & $M$ & $D E$ & $\alpha$ & Asimetria & Curtosis & Normailidad \\
\hline Estrategias metacognitivas & & & & & & \\
$\quad$ Elaboración de relaciones comprensivas & 4.7 & 0.78 & .801 & 0.250 & -0.247 & .07 \\
$\quad$ Organización de la información: Esquemas & 4.4 & 1.34 & .784 & -0.028 & -0.196 & .29 \\
$\begin{array}{l}\text { Organización de la información: Ideas } \\
\text { principales }\end{array}$ & 3.0 & 1.00 & .618 & -0.575 & -0.540 & .04 \\
$\quad$ Selección de la información & 4.6 & 0.70 & .700 & -0.093 & 0.847 & .08 \\
Supervisión y autoevaluación & 5.1 & 0.93 & .799 & -0.226 & -0.251 & .22 \\
$\quad$ Planificación del aprendizaje & 4.4 & 0.81 & .778 & -0.259 & 0.141 & .20 \\
Motivación & & & & & & \\
Ansiedad y miedo al fracaso & 4.0 & 0.80 & .635 & 0.425 & 0.625 & .22 \\
Concentración y distracciones & 4.4 & 0.77 & .605 & 0.332 & 1.385 & .04 \\
Motivos para estudiar & 5.2 & 0.77 & .645 & -0.463 & -0.248 & .02 \\
Ambiente social & 4.7 & 0.74 & .596 & -0.164 & -0.721 & .16 \\
Estilos de aprendizaje & & & & & & \\
Activo & 13.8 & 2.64 & .437 & -0.258 & 0.005 & .10 \\
Reflexivo & 14.5 & 2.83 & .546 & -0.354 & -0.260 & .04 \\
Teórico & 13.7 & 2.78 & .498 & -0.179 & -0.547 & .04 \\
Pragmático & 13.5 & 2.69 & .457 & -0.055 & -0.501 & .12 \\
\hline
\end{tabular}


Al considerar los estilos de aprendizaje (ver Tabla 1), se observa, según la interpretación de Alonso, Gallego y Honey (1999) y con un baremo definido por los participantes, que el $46 \%$ de los estudiantes no tiene un único estilo dominante. El estilo que se presenta con mayor frecuencia es el teórico $(21 \%$ de los estudiantes), seguido del reflexivo (14\%) y de los estilos activo y pragmático, con una frecuencia muy similar $(10 \%)$.

El modelo planteado para analizar la relación entre las variables latentes - estrategias metacognitivas, motivación y estilos de aprendizaje- alcanza niveles de ajuste satisfactorios (Hair, Black, Babin y Anderson, 2010) en tres de los índices (ver Figura 1): chi cuadrada normada menor que 3 , CFI mayor que .9 y RMSEA menor que .08. El GFI no supera el .90 y la chi cuadrada resulta significativa.

En el modelo de medida se puede observar que la motivación queda definida principalmente por la concentración sin distracciones y la ansiedad o miedo al fracaso. Sin embargo, presentan signos contrarios, dando a entender que son inversas una con respecto a la otra en su aporte al definir la motivación. Con las estrategias metacognitivas se interpreta directamente, a mayor puntaje en las variables observadas, mayor uso de estrategias metacognitivas, con excepción de la organización de la información en ideas principales. En los estilos se observan todas las cargas con los mismos signos indicando que no hay estilos opuestos entre ellos.

En el modelo de estructura se observa una correlación muy importante entre la motivación y las estrategias metacognitivas $(\phi=.88)$. Se puede decir que a mayor motivación se asocia mayor uso de estrategias metacognitivas e inversamente. Ahora bien, la relación entre estos dos constructos y los estilos de aprendizaje es muy baja e inclusive no significativa $(p>.05)$, tanto la relación entre los estilos de aprendizaje y la motivación $(r=.09)$, como la relación con las estrategias metacognitivas $(r=.14)$.

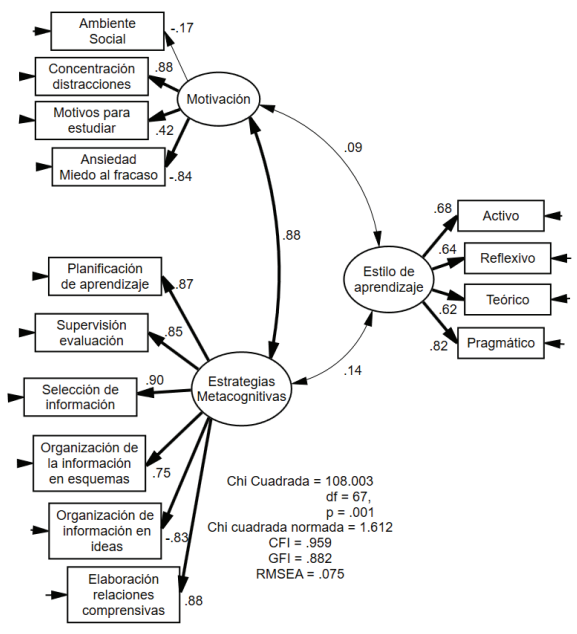

Figura 1. Modelo de relación entre la motivación, estrategias metacognitivas y los estilos de aprendizaje. Las líneas gruesas indican relaciones significativas $(p<.05)$.

\section{Discusión}

En este estudio se encontró que las estrategias metacognitivas que tienden a ser regularmente utilizadas son la supervisión y la autoevaluación, en tanto que la organización de la información presenta el más bajo desempeño, presumiblemente debido a la ausencia de un entrenamiento adecuado. Esta presunción se basa en que varios autores (Collins, 1994; Sperling, Walls y Hill, 2000; Tei y Stewart, 1985) consideran que la metacognición es la conciencia de que se posee un control comprensivo del conocimiento, lo cual permite su empleo 
apropiado. En la misma línea, Hacker (1995) afirma que el conocimiento de su propio conocimiento, procesos y estados cognoscitivos y afectivos faculta al sujeto para supervisarlos y regularlos deliberadamente. Valdría la pena realizar más investigaciones longitudinales en poblaciones de nivel educativo medio, con el fin de analizar más a profundidad la maduración cognitiva en el ser humano, sus motivaciones y estilos de aprendizaje a lo largo de los años. Teniendo en cuenta los resultados del estudio, se recomienda aumentar en los contextos escolares el uso de estrategias que promuevan la motivación escolar, ya sea a través de incentivos académicos como notas, reconocimiento social, acceso a espacios culturales y deportivos, etc., así como el aumentar el uso de estrategias metacognitivas y cognitivas que faciliten el aprendizaje autorregulado.

En esta investigación se encontró también una relación significativa fuerte $(\phi=.88)$ entre los constructos motivación y estrategias metacognitivas. Este hallazgo coincide con la literatura vigente (Alarcón Díaz, 2013; Herczeg y Lapegna, 2010; Núñez Alonso et al., 2006; Pan et al., 2013; Paris y Winograd, 2001 Suárez Riveiro y Fernández Suárez, 2013). Esta relación, que ha sido muy estudiada últimamente, estaría señalando que los estudiantes motivados son capaces de desarrollar estrategias metacognitivas que favorecen su propio aprendizaje.

Respecto a la variable motivación dentro de las características de los estudiantes evaluados, en el presente trabajo se destacan los motivos para estudiar, la ansiedad y el miedo al fracaso. Los motivos para estudiar cobran valor en el momento del desarrollo de los estudiantes evaluados; para la edad escolar de un adolescente, la construcción de expectativas en torno de las acciones que ejecuta es determinante. El joven logra de manera continua construir ideas en relación con lo que puede obtener a corto o largo plazo mediante el estudio u otra actividad en la cual invierte gran parte de su tiempo. El valor de la expectativa se contrasta con la propuesta de Morales Rodríguez (2011), quien plantea que la expectativa se convierte en el orientador de la conducta y permite connotar el valor que la persona asigna a la meta. Para los jóvenes evaluados, las actividades que realizan en su contexto académico son el medio para acceder a experiencias y aprendizajes posteriores.

En relación con el miedo al fracaso, es probable que los estudiantes se esfuercen y persistan en la obtención de resultados académicos satisfactorios (Suárez Riveiro y Fernández Suárez, 2013). La motivación se convierte en un orientador de la conducta de los estudiantes, estimulándolos a estar más atentos, activos y a adaptar sus estrategias de estudio en función de las actividades que realizan, con el propósito de controlar los resultados de sus actividades. El concepto de locus de control interno y externo puede ayudar a explicar los índices motivacionales de los estudiantes evaluados, especialmente en el aspecto que aquí se discute; si el locus de control hace referencia a la creencia que tiene el estudiante en relación con la procedencia del control de sus actividades, atribuir control de las actividades y sus resultados a él mismo y no a los aspectos ambientales que le rodean puede contribuir positivamente al mejoramiento de sus resultados académicos, en tanto los estudiantes logren evaluar los recursos con los que cuentan y ajustar sus estrategias en el cumplimiento de las tareas. 
El ambiente social juega un papel importante en la motivación de los estudiantes, pues las relaciones con las demás personas y las variables que se encuentran en su ámbito escolar logran activar los recursos cognitivos, emocionales y comportamentales que permiten llegar al objetivo que se han planteado. Es posible explicar la influencia del ambiente social a partir del concepto de motivación extrínseca. La fuente motivacional procede del ambiente del estudiante, quien se ve mediado por el reconocimiento social, la obtención de logros académicos materializados en la necesidad de aprobar o el interés por obtener buenas calificaciones. También puede relacionarse con aspectos ambientales del proceso educativo, como las estrategias pedagógicas que aplica el docente, en las cuales el estudiante encuentra formas de reconocimiento social y académico (López Aguado y Silva Falchetti, 2009).

En este estudio se indagan también las características motivacionales de los estudiantes en relación con otro constructo que son los estilos de aprendizaje. Se identificó que la puesta en práctica de un estilo de aprendizaje concreto no está determinada tanto por el objetivo que el alumno busca conseguir cuando se enfrenta a una tarea de aprendizaje (superarla o conseguir un aprendizaje) como con las estrategias que activa para resolverla (López Aguado y Silva Falchetti, 2009). Lo anterior indica una aparente ausencia de relación entre la motivación y los estilos de aprendizaje y en ese sentido los resultados del presente estudio coinciden con los de dichos autores.

Respecto a la relación entre estrategias metacognitivas y estilos de aprendizaje, en la literatura se ha encontrado que estudiantes con ciertos estilos de aprendizaje, tienden a tener una preferencia por determinadas estrategias metacognitivas (Arias Gallegos et al., 2014; Cázares Castillo, 2009; Gravini Donado y Iriarte Diazgranados, 2008). Además, se ha encontrado un impacto positivo sobre el desempeño de ciertas actividades académicas en los estudiantes con ciertos estilos de aprendizaje que hacen uso de determinadas estrategias metacognitivas (Pujol, 2008). En la presente investigación no se observó esa correlación, lo cual puede atribuirse a que en esta investigación el desarrollo de los diferentes estilos de aprendizaje es muy equilibrado, además de que únicamente la mitad de los encuestados tiende a tener un estilo preferente. Otro factor que podría estar afectando es el de la edad, pues en la mayoría de los otros estudios la relación se ha encontrado en estudiantes universitarios. En conclusión, los estilos de aprendizaje conforman un constructo que es independiente de la motivación y las estrategias metacognitivas.

\section{Referencias}

Aguilera Pupo, E. y Ortiz Torres, E. A. (2008). La caracterización de perfiles de estilos de aprendizaje y sus implicaciones didácticas en la educación superior. Pedagogía Universitaria, 13(5), 1-13.

Alarcón Díaz, M. A. (2013). Motivación, estrategias de aprendizaje y metacomprensión lectora: un estudio descriptivo en alumnos universitarios peruanos. Revista Educación y Desarrollo Social, 7(1), 71-78. https://doi.org/ $10.18359 /$ reds. 735

Alonso, C., Gallego, D. y Honey, P. (1999). Los estilos de aprendizaje ( $5^{\mathrm{a}}$ ed.). Bilbao: Mensajero.

Arias Gallegos, W. L., Zegarra Valdivia, J. y Justo Velarde, O. (2014). Estilos de aprendizaje y metacognición en estudiantes de psicología de Arequipa. Liberabit, 20(2), 267-279.

Blumen, S., Rivero, C. y Guerrero, D. (2011). Universitarios en educación a distancia: estilos de aprendizaje y rendimiento académico. Revista de Psicología, 29(2), 225-243. 


\section{MOTIVACIÓN PARA EL TRABAJO INTELECTUAL}

Cázares Castillo, A. (2009). El papel de la motivación intrínseca, los estilos de aprendizaje y estrategias metacognitivas en la búsqueda efectiva de información online. Pixel-Bit, 35, 73-85.

Contreras Gastélum, Y. I. y Lozano Rodríguez, A. (2012). Aprendizaje auto-regulado como competencia para el aprovechamiento de los estilos de aprendizaje en alumnos de educación superior. Revista Estilos de Aprendizaje, 10(5), 114-147.

Collins, N. D. (1994). Metacognition and reading to learn. ERIC Digest. Recuperado de http://www .indiana.edu/ reading/ieo/digests/d96 .html

Gravini Donado, M. L. e Iriarte Diazgranados, F. (2008). Procesos metacognitivos de estudiantes con diferentes estilos de aprendizaje. Psicología desde el Caribe, 22, 1-24.

Hacker, D. (1995). Metacognition: Definitions and empirical foundations. New York: Roudlegde.

Hair, J., Black, W., Babin, B. y Anderson, R. (2010). Multivariate data analysis ( $7^{\mathrm{a}}$ ed.). México: Pearson Prentice Hall.

Herczeg, C. y Lapegna, M. (2010). Autorregulación, estrategias y motivación en el aprendizaje. Lenguas Modernas, 37, 9-19.

Isaza Valencia, L. (2014). Estilos de aprendizaje: una apuesta por el desempeño académico de los estudiantes en la educación superior. Encuentros, 12(2), 25-34.

Kohler Herrera, J. L. (2013). Rendimiento académico, habilidades intelectuales y estrategias de aprendizaje en universitarios de Lima. Liberabit, 19(2), 277-288.

Lamas Rojas, H. (2008). Aprendizaje autorregulado, motivación y rendimiento académico. $\mathrm{Li}$ berabit, 14, 15-20.

Lanz, M. (Comp.). (2006). El aprendizaje autorregulado. Enseñar a aprender en diferentes entorno educativos. Buenos Aires: Noveduc.

Laudadío, M. y Da Dalt, E. (2014). Estudio de los estilos de enseñanza y estilos de aprendizaje en la universidad. Educación y Educadores, 17(3), 483-498. doi:10.5294/edu.2014.17.3.5

López Aguado, M. y Silva Falchetti, E. (2009). Estilos de aprendizaje. Relación con motivación y estrategias. Revista Estilos de Aprendizaje, 4(2), 36-55.

Maggiolini, L. M. (2013). Estrategias de motivación en una era digital: Teléfonos móviles y Facebook en el aula. Digital Education Review, 24, 83-97. Recuperado de http://revistes .ub.edu/index.php/der/article/view/11278

Morales, Rodríguez, F. M. (2011). Aprendizaje, motivación y rendimiento en estudiantes de lengua extranjera inglesa. Psicología Educativa, 17(2), 195-207. https://doi.org/10.5093/ ed2011v17n2a6
Núñez Alonso, J. L., Lucas, J. M. A., Navarro Izquierdo, J. G. y Grijalbo Lobera, F. (2006). Validación de la Escala de Motivación Educativa (EME) en Paraguay. Revista Interamericana de Psicología, 40(3), 391-398.

Pan, I., Regueiro, B., Ponte, B., Rodriguez, S., Piñeiro, I. y Valle, A. (2013). Motivación, implicación en los deberes escolares y rendimiento académico. Aula Abierta, 41(3), 13-22.

Paris, S. G. y Winograd, P. (2001). The role of self-regulated learning in contextual teaching: principles and practices for teacher preparation. Recuperado de http://eric.ed.gov/?id= ED479905

Pool-Cibrián, W. J. y Martínez-Guerrero, J. I. (2013). Autoeficacia y uso de estrategias para el aprendizaje autorregulado en estudiantes universitarios. Revista Electrónica de Investigación Educativa, 15(3), 21-37. Recuperado de https://redie.uabc.mx/redie/article/view/551/ 810

Pujol, L. (2008). Búsqueda de información en hipermedios: efecto del estilo de aprendizaje y el uso de estrategias metacognitivas. Investigación y Postgrado, 23(3), 45-67.

Rinaudo, M., Chiecher, A. y Donolo, D. (2003). Motivación y uso de estrategias en estudiantes universitarios. Su evaluación a partir del Motivated Strategies Learning Questionnaire. Anales de Psicología, 19(1), 107-119.

Rodríguez, S., Piñeiro, B., Regueiro, E., Gayo, E. y Valle, A. (2014). Metas académicas, estrategias de aprendizaje y rendimiento académico en educación secundaria. Magister, 26, 1-9. https:// doi.org/10.1016/S0212-6796(14)70012-X

Rosário, P., Pereira, A., Högemann, J., Nunes, A. R., Figueiredo, M., Núñez, J. C. . . . Gaeta, M. L. (2014). Autorregulación del aprendizaje: una revisión sistemática de revistas de la base SciELO. Universitas Psychologica, 13(2), 781-798. doi:10.11144/Javeriana.UPSY13-2 .aars

Sanfabián Maroto, J. L., Belver Domínguez, J. L. y Álvarez Álvarez, C. (2014). ¿Nuevas estrategias y enfoques de aprendizaje en el contexto del Espacio Europeo de Educación Superior? Revista de Docencia Universitaria, 12(4), 249280. https://doi.org/10.4995/redu.2014.5623

Sperling, R., Walls, R. y Hill, L. (2000). Early relationships among self-regulatory constructs: Theory of mind and preschool children's problem solving. Child Study Journal, 30(4), 233253.

Suárez Riveiro, J. M. y Fernández Suárez, A. P. (2013). Un modelo sobre cómo las estrategias motivacionales relacionadas con el componente de afectividad inciden sobre las estrategias 
cognitivas y metacognitivas. Educación XX1, 16(2), 231-246. https://doi.org/10.5944/ educxx 1.16.2.2641

Suárez Riveiro, J. M., Fernández Suárez, A. P., Rubio Sánchez, V. y Zamora Menendez, A. (2016). Incidencia de las estrategias motivacionales de valor sobre las estrategias cognitivas y metacognitivas en estudiantes de secundaria. Revista Complutense de Educación, 27(2), 421-435.

Tei, E. y Stewart, O. (1985). Effective studying from text: Applying metacognitive strategies. Forum for Reading, 16(2), 46-55.

Torrano Montalvo, F. y González Torres M. (2004). $\mathrm{El}$ aprendizaje autorregulado: presente y futuro de la investigación. Revista Electrónica de Investigación Psicoeducativa, 2(1). http://dx.doi .org/10.25115/ejrep.3.120

Tripodoro, V. A. y De Simone, G. G. (2015). Nuevos paradigmas en la educación universitaria: Los estilos de aprendizaje de David Kolb. $\mathrm{Me}$ dicina, 75(2), 109-112.
Valle, A., Rodríguez, S., Núñez, J., Cabanach, R. G., González-Pienda, J. A. y Rosario, P. (2010). Motivación y aprendizaje autorregulado. Revista Interamericana de Psicología, 44(1), 86-97.

Vázquez, S. M., Noriega Biggio, M. y García, S. M. (2013). Relaciones entre rendimiento académico, competencia espacial, estilos de aprendizaje y deserción. Revista Electrónica de Investigación Educativa, 15(1), 29-44. Recuperado de http://redie.uabc.mx/redie/article/ view/328/510

Wichadee, S. (2013). Facilitating students' learning with hybrid instruction: A comparison among four learning styles. Electronic Journal of Research in Educational Psychology, 11(1), 99116. doi:10.25115/ejrep.v11i29.1559

Recibido: 4 de febrero de 2019 Revisado: 17 de marzo de 2019 Aceptado: 27 de mayo de 2019 